\title{
VISUAL ANALYTICS FOR EVALUATION OF VALUE IMPACT IN ENGINEERING DESIGN
}

\author{
Timoleon Kipouros $^{1}$ and Ola Isaksson ${ }^{2}$ \\ ${ }^{1}$ University of Cambridge, Department of Engineering, \\ Trumpington Street, Cambridge, CB2 1PZ, UK \\ tk291@eng.cam.ac.uk \\ ${ }^{2}$ GKN Aerospace Engine Systems, R\&T, \\ 46181, Trollhättan, Sweden \\ ola.isaksson@gknaerospace.com
}

Keywords: Engineering Design, Computational Design, Interactivity, Parallel Coordinates, Aerospace, Decision Making, Value Assessment, Value Driven Design, Visual Analytics.

\begin{abstract}
Many traditional engineering design processes in industry have evolved, and nowadays they are supported by computational tools and methods. As a consequence, apart from the quantity of information and data, a network of simulation methods produces information at various level of detail and nature. Furthermore, human interactivity is an essential consideration when building and designing such computational systems, simply because there is a requirement to process and understand this information that is produced during an engineering project effectively in real time.
\end{abstract}

We introduce here a Visual Analytics perspective in computational engineering design process, as well as tightening the interactive analysis of engineering data with Parallel Coordinates and Scatter plots. To demonstrate the benefits of the proposed approach we use a case study that describes the design of an aero engine component critically suffering from the operating conditions but at the same time from the change of specifications and customer requirements.

Some of the benefits include minimisation of rework through early identification of behaviour in selected Value Dimensions, as well as the ability to trade product performance (e.g. weight, minimum expected life) with internal stakeholder expectations (e.g. higher overall productivity of aircraft, low degree of aircraft modifications, faster design convergence). Furthermore, the proposed method develops the ability to identify architectural options that align with Value Creation Strategies, but also the evaluation of design options in advance of physical trade studies. 


\section{INTRODUCTION}

These days, people perceive for granted complex engineering products, such as an aircraft, and most importantly use them in their daily routine. As a result, the requirements and specifications should meet the expectations of the customers and stakeholders simultaneously. In other words, the technical and engineering objectives that usually drive a design process, should now reflect the impact of customer satisfaction. In our example, a passenger would wish to have more leg space for comfort, better quality of air in the cabin, and cheap ticket price, and expect to be able to reach at the airport and spend minimum amount of time during security control and boarding. But how do these expectations translate to engineering performance indicators? How these metrics can be modelled? What are the relationships between such properties? We believe Visual Analytics and Big Data management can assist toward finding answers to these questions, but more importantly to create new questions that weren't thought before.

In an engineering design process, as illustrated in Figure 1, we start with the description of a problem, which we then need to formulate and model, often, in a computational/simulation environment. The same problem can have many model instances depending on the level of detail that is described, or even the point of view of the analysis. Each of these problem formulations are then explored with design optimization, analysis tools, or similar. Before we are able to cascade this information downstream in the process for decision making, we need to collect, synthesize, and process the data.

The process to collect this data, most of the times, has a complex structure, since many different analysis and simulation tools are used during an engineering design project. Furthermore, in many cases they are located in geographically different sources.

The mere amount of aspects that need to be considered simultaneously when making decisions during product development is one of the main reasons why synthesis in engineering design relies on experienced and advanced human cognitive skills (ref needed). Since the introduction of computational support, the ability to design and optimize products have made several leaps forward. As one example, the actual definition and representation of a compressor-or turbine- blade is no longer possible to define by hand. Advances in Computational Fluid Dynamics and the following ability to optimize such designs have enabled efficiency of compressor design to advance from $9 \mathrm{X} \%$ to over $99 \%$ in just a couple of decades. A second aspect is that to differentiate on the marketplace, success is increasingly determined by how well the product perform from multiple perspectives.

From an industrialist's perspective, we are now at a point where we no longer create a single concept with small variations of forthcoming products, but rather exploring entire design spaces using advanced computer tools. Engineers have the capability to produce more data per instance, for more variants and for more situations than ever before.

There is a need to visualize the data and more importantly to be able to interpret and understand what these data mean in a sensible way. Traditional ways of the representation of the behavior of a design solution include the use of animations of e.g. deflection and distortion of a product (modal analysis, stress plots, etc.). Where the engineer could display results of one, or few alternatives, now is no longer feasible due to the multitude of variants and circumstances (loads) that the product is exposed to. Many aspects need to be explored for a range of alternative solutions. As a consequence, even if it is possible to generate such information, the analysis of a multi-parameter design space for many alternatives drive the need to interact and understand much richer and more complex dataset

We need to complement other visualization techniques to enable rich understanding of many alternatives subject to variation to many variables or aspects. This is also another major contribution of this paper. 


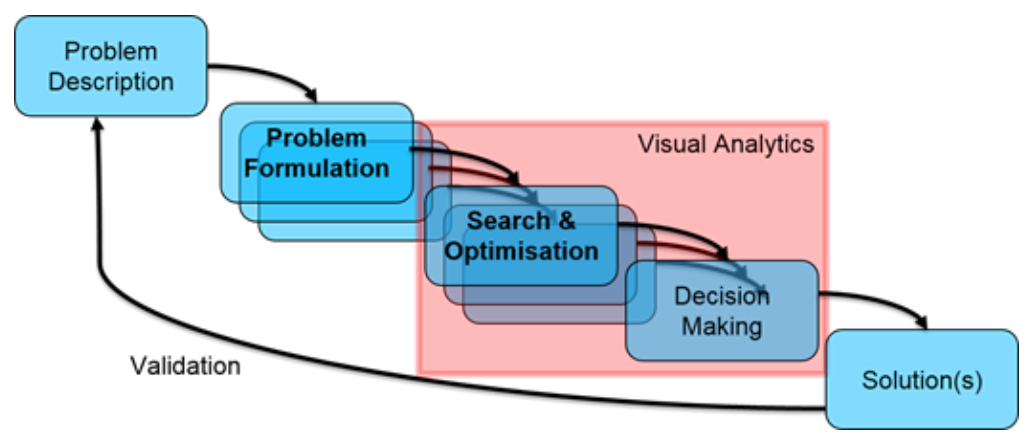

Figure 1: High level view of an engineering design process supported by visual analytics tools and methods.

\section{BACKGROUND AND LITERATURE}

Decision making during development is by nature multi-disciplinary, and several authors have proposed ways on how to explore design spaces in many dimensions. The MOVA framework by Woodruff et.al. [6] extended a finite dimensional design strategy to a more general framework to include multiple objectives, and compared the approach with alternative decision making strategies. Keim et al [15] summarized the area of Visual Analytics as a result from a three year European project, and concluded that assisting designers using visual analytic systems was one of the key challenges for the future.

In Figure 2 it is shown a schematic representation of a concept of how to facilitate human interactivity within the automated computational engineering design cycle.

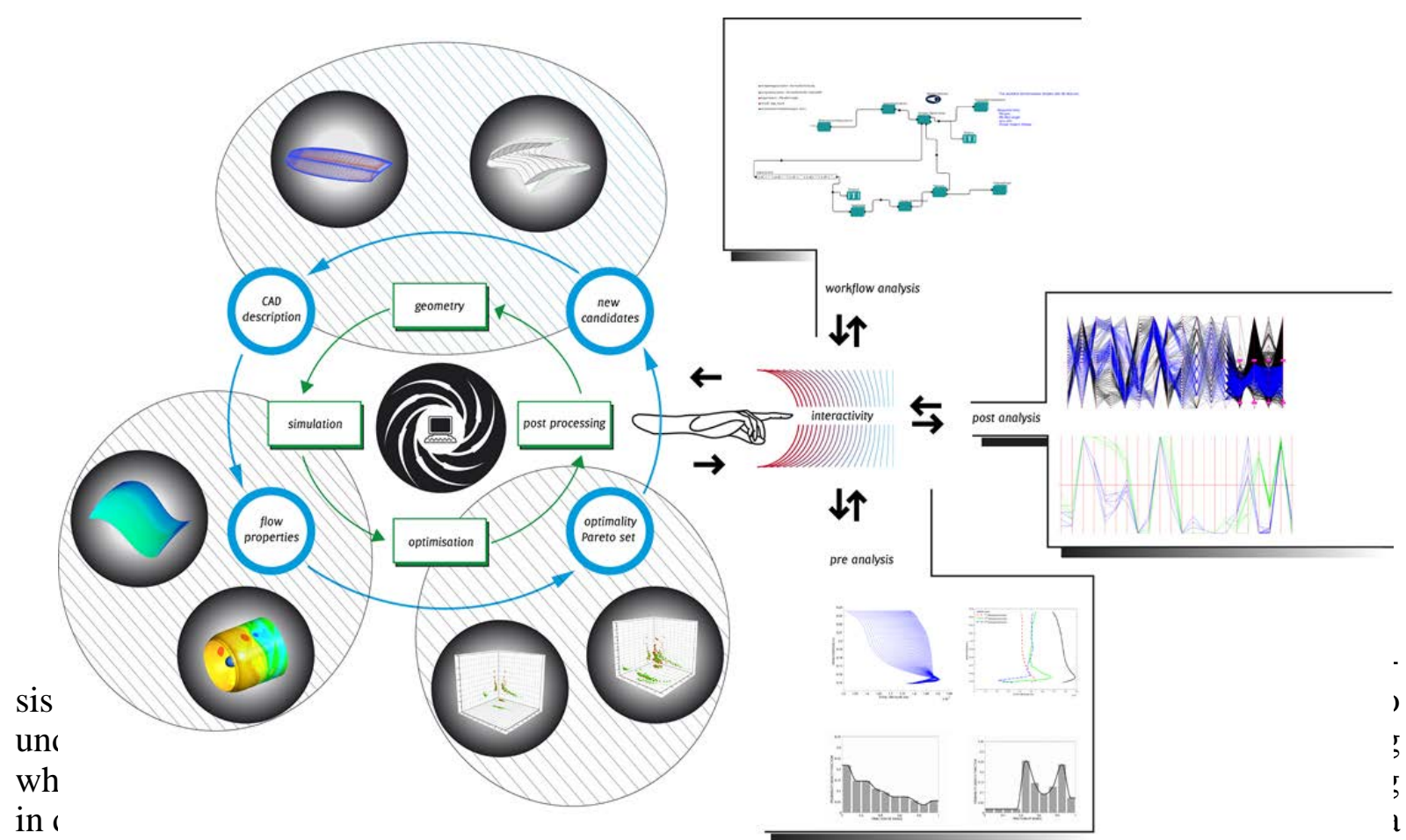

of Value Driven Design previously [16]. It has further been demonstrated the effect of contextual information of product oriented data and observed the effect on engineering team attention to how data was visualized [14]. The ability to interact with alternatives and changes conditions, such as product requirements changes and/or preference changes are clearly emphasized. 
Engineering Design is a highly iterative activity, where the definition of forthcoming products is typically matured through managing iteration and changes. Any decision support need to support the study of variation, trading, etc. Data is typically produced in engineering tools, but also in less quantitative manner. Subjective metrics such as confidence, appearance, risk, etc co-exist with quantifiable aspects such as stresses, strains etc.

Parallel coordinates (\|-coords) can support and facilitate the possibility to identify trends and relationships between technical properties and specification characteristics in a multidimensional domain.

There are platforms and frameworks under investigation and development that facilitate the human interaction and drive/guide optimization studies. But also offer visual representation of the product too in an additional engineering analysis environment (i.e. Workways [12]).

\section{AN ENGINEERING EXAMPLE}

The initial aim and objective of this work was to identify the means to connect high level stakeholder and customer expectations and requirements to actual technical key performance characteristics and indicators. We use the Value Assessment method as introduced in [2] and expanded in [1]. Here, we only provide a brief description of the main stages of the Value Assessment process. For more details the reader should refer to [1] and [2].

\subsection{Value Driven Design Methodology}

A Value Assessment process can be best described within the Value Driven Design process as shown in Figure 3.

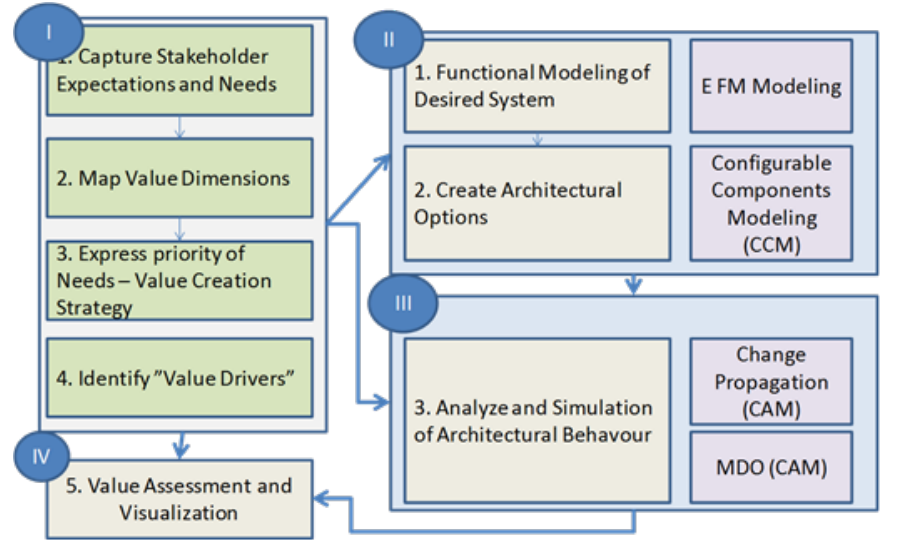

Figure 3: The four phases of the Value Driven Design methodology.

In phase I the stakeholder expectations and needs are captured and linked with Value Dimensions and Value Drivers. The often tacitly expressed expectations are organized into a Value Creation Strategy (VCS), this is the prioritized set of stakeholder needs that can be influenced in design via the Value Drivers. The Value Drivers are consequently aspects wherein it is possible to define the design parameters to explore. Typically, the breath of information in phase I is vast. Explicit and numerically defined expectations, such as expected range or target weights are mixed with tacit and ill-defined expectations, such as being "sustainable" or "easily maintainable”, “easy to integrate into a system” etc. Explicit and quantifiable modeling may not be feasible, not at least in early phases, but may still be necessary to include in decision making.

The second phase takes the VCS as input and serve to support the design synthesis phase. Searching for solutions in the design space is divided into an architectural modeling phase, in this case using an extended function means modeling approach, where the functionality of the 
systems is modeled and the alternative design solutions are defined. At this phase, the characteristics of the forthcoming design is modeled for all relevant candidate design solutions. One way of representing an architectural option is via Design Structure Matrices (DSM's), where the pattern of internal dependencies of design objects provide a quantifiable pattern of the architectural option.

The third phase introduce analytical tools to analyze the behavior of the alternative architectures along the evaluation dimensions identified as Value Dimensions in phase I. One efficient method to understand the behavior of the Change Propagation method [], where dependencies between the architectural objects are assigned with probability and impact information. Change propagation analysis then generate information of how an architectural definition react to changes and perturbations. It is possible to define first order relation to several stakeholder needs, such as "integration ability" and "development process efficiency" [].

In the fourth phase is the focus in this paper and organizes the results from the simulated behavior of all alternative architectures studies. Using II-coordinates as a means of visualization, the experience and intuition of the designer can be combined with the patterns generated using analytical methods. Since multiple aspects and multiple architectural options are studied, the pattern recognition abilities of humans is used to facilitate interactive exploration and search for suitable combinations of input and output.

\subsection{A Brief Description of the Case Study}

The method using visual analytics is applied onto a case study. The product studied is a sub system of an aircraft jet-engine, a rear turbine structure (RTS). From an applied perspective, the RTS is a tightly integrated structural component of the jet engine used to propel the aircraft and provide power to the aircraft also for other purposes. The scenario is that of a reengine scenario where the aircraft manufacturer wish to upgrade the performance of the aircraft by replacing and/or upgrading the existing engine type. The engine manufacturing consortia consisting of the jet engine OEM and its design partners need to understand the new consequences and assess what design options exists that are needed to satisfy the ambitions of the re-engine scenario.

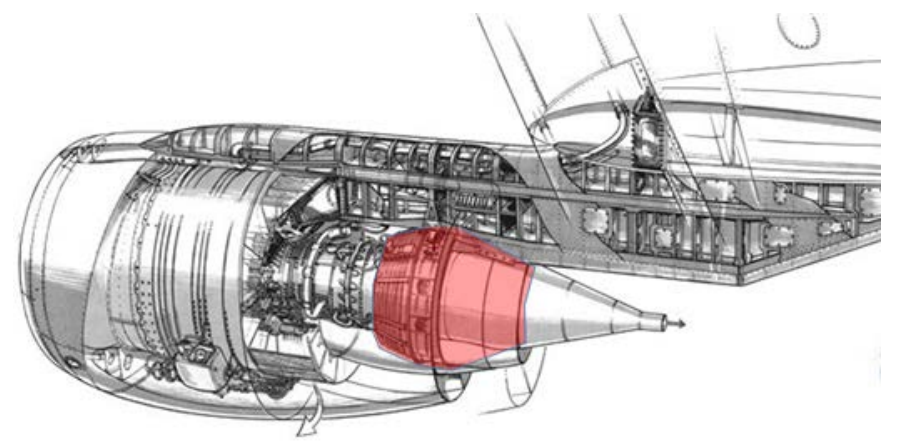

Figure 4: Re-engine scenario: turbine exit structure (illustration from Flight Magazine).

In the European project TOICA, the scenario is defined as a context, wherein the Value Assessment Methodology, described in [1] and introduced above has been followed. In this paper we focus on phase IV but capture portions of the dataset for clarification and to present the sources of information used when assessing value in the interactive way using parallel coordinates. From phase I, a range of Value information is captures, each linking Stakeholder Expectations to Value Drivers. In Figure 5 this is illustrated for the "Higher Productivity of Aircraft” Stakeholder Expectations. This expectation eventually is influenced by weight since 
this has a $1^{\text {st }}$ order influence on payload, and minimum expected life which has an equal direct influence in the operating cost of an aircraft.

\begin{tabular}{|c|c|c|c|}
\hline $\begin{array}{l}\text { Stakeholder } \\
\text { Expectations }\end{array}$ & $\begin{array}{l}\text { Stakeholder } \\
\text { Needs }\end{array}$ & $\begin{array}{l}\text { Value } \\
\text { Dimensions }\end{array}$ & Value Drivers \\
\hline \multirow{2}{*}{$\begin{array}{l}\text { Higher } \\
\text { productivity of } \\
\text { aircraft }\end{array}$} & $\begin{array}{l}\text { Increased } \\
\text { payload }\end{array}$ & \multirow{2}{*}{$\begin{array}{l}\text { Mission } \\
\text { performance }\end{array}$} & Weight \\
\hline & $\begin{array}{l}\text { Reduced } \\
\text { operating cost }\end{array}$ & & $\begin{array}{l}\text { Minimum } \\
\text { expected life }\end{array}$ \\
\hline
\end{tabular}

Figure 5: Expression of generic stakeholder expectations and needs, and the mapping to value dimensions and value drivers.

Since the stakeholder expectation of "Higher productivity of Aircraft" and both of its Stakeholder Needs are attributed to the operator of the aircraft, the value dimension is set to "Mission Performance" as a classification. Notably, there are other Value Dimensions, addressing other aspects of stakeholders, such as how costly the products are to integrate into a system etc.

The weight of a component a good example of a Value Driver that can be assigned a firm and quantifiable target. It can equally be related to the impact on productivity of the aircraft since and it is frequently the case in aerospace that weight is given a direct relation to productivity of the aircraft. In design phases, it is equally common to formulate penalty relations in monetary terms if target weights are not met.

In a similar manner, there are relations with the expected life and the productivity of operating the aircraft. Maintenance schedules, repair and inspection costs are directly related to the satisfaction of a minimum expected life.

Both minimum expected life and weight of components are examples of quantifiable value drivers. To evaluate weight and expected life, there is effectively no mature way of analyzing this impact for new alternative designs without modeling the CAD definition (gives the volume) and making physics based evaluations using computational techniques such as finite element analysis (give the stresses and strains) and some lifing analysis technique (such as crack propagation and/or fatigue analysis).

The actual relations between weight and performance, or minimal life and operating cost are business sensitive data and not disclosed explicitly in this paper.

Phase II, the functional modeling is conducted using a functions means modeling approach. Alternative design solutions for functions are identified and constraints and dependencies between the design solutions are captured. The method is described in more detail in [17] and is implemented in a modeling tool - CCM (Configurable Components Modeler). In this way, alternative architectures of the component can be represented as Design Structure Matrices' (DSM’s).

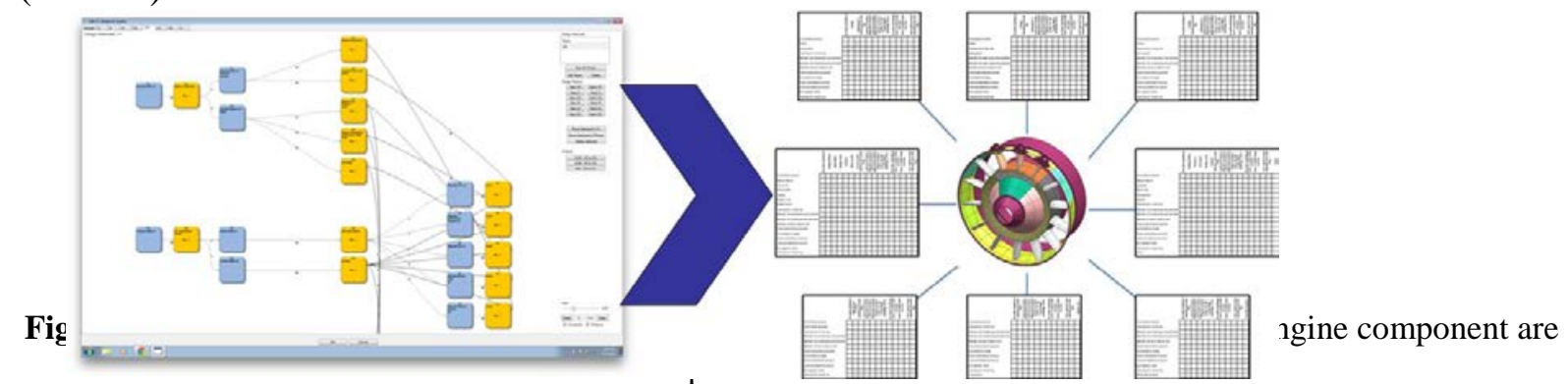

In the third step, the behavior of each alternative architecture is analyzed using a Change Propagation Method (CPM) [18] using CAM (Cambridge Advanced Modeler). 


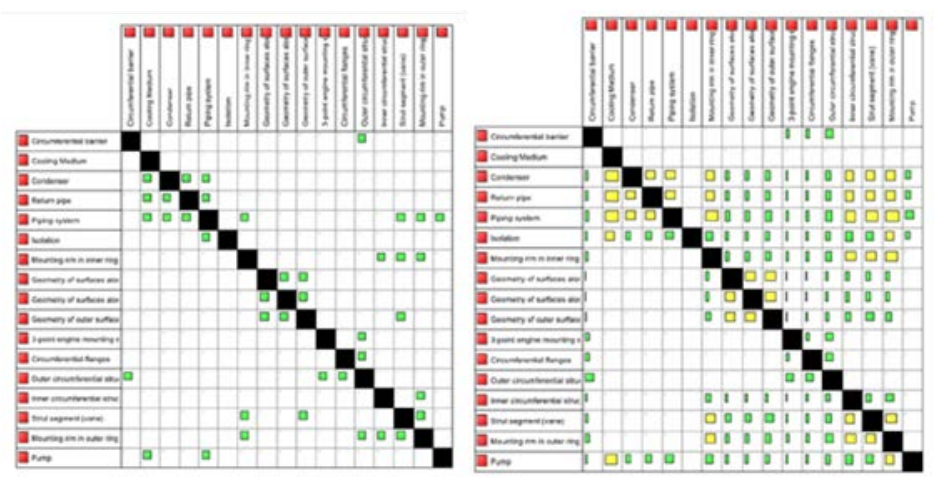

Figure 7: Through Function-Means modeling in CCM, alternative architectures of a RTS engine component are exported as DSM's.

The CPM analysis allow analysis that link the behavior of the architecture to the Stakeholder Needs and Value Dimensions. One value dimension is "Integration ability", wherein stakeholder expectations and needs such as "ability to insert into an existing aircraft and engine system". Relating the number of connections between design solutions can be related directly to the effort required to integrate into a system. A higher number of connection is assumed to require a larger effort in integration.

\section{INTERACTIVE ANALYSIS WITH PARALLEL COORDINATES}

\subsection{Methodology}

The input to the interactive analysis for Value Assessment are initially the results from the functional analysis and CPM analyses. Parallel coordinates are used to display simultaneously the results onto each value dimensions in the same plot as the design variables. The architect can work interactively with the data set, and filter out architectures in several ways, either via filters on the parameters or via a graphical plot in 2D where two selected parameters are compared to each other. As the concepts are refined, more advanced modeling and analysis tools can be used to predict the behavior or the concept.

The first step (to the left below) operate on the pre-embodiment DSM data. In this steps alternative variants are down selected, and the most promising variants are selected for further refinement. Selection of variants allow physical and parametric geometrical modeling and finite element analyses to be conducted. The same tool and interactive analysis is re-used with a richer data set. This is described to the right in the figure below.

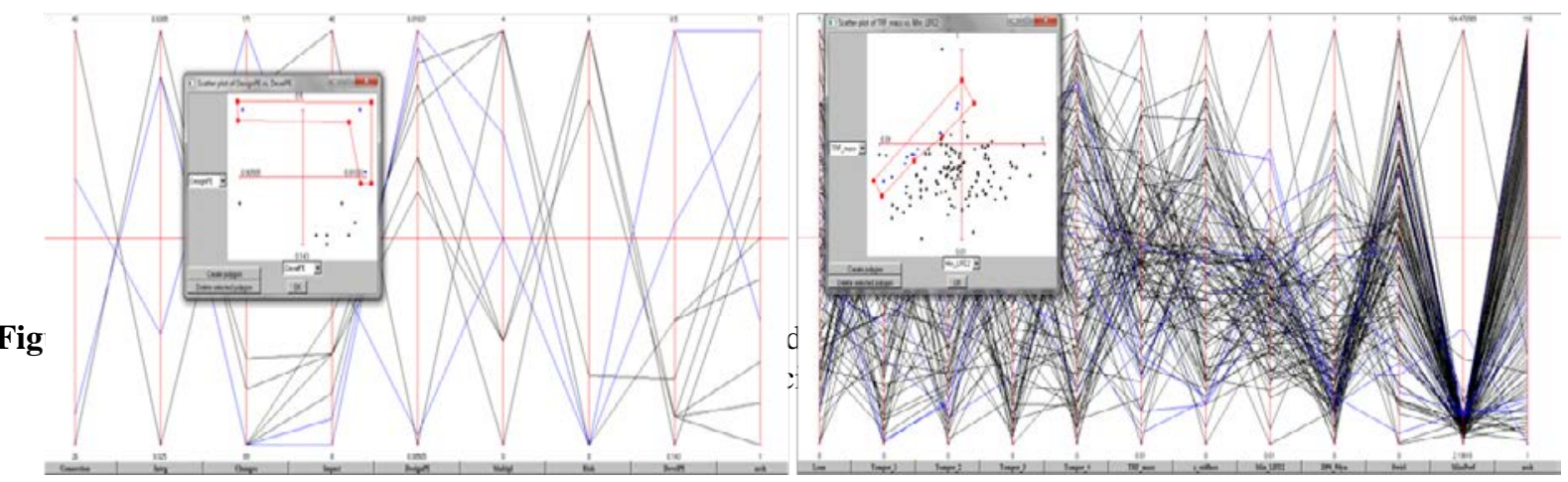

\section{FUTURE WORK AND CONCLUSIONS}

The approach presented, and the interactive way of analyzing datasets was used to analyse initial architectural descriptions based on DSM representations and CPM as analysis method. The same visual analytics tools is re-used as the data set is enriched through more detailed and 
refined models of the architectures. In particular, the visual and interactive tool has been demonstrated effective as a means to facilitate the communication between architects and different engineering design teams. The ability to link "high level" objectives through the Value Creation Strategy, to the design variables characterizing the various architectures has been demonstrated successful. Since information in complex products during development typically engage many disparate competences and specialist teams, the value of communicating expectations and technical results are decisive. The Visual Analytics methods and tools are highly suitable to combine data from different sources and from different nature.

In particular, the ability to analyse the often extensive data sets by filtering, selecting and searching for combinations of parameter values is an important feature. This is typically the task for multi disciplinary optimization specialists, whereas though the visual analytics approach different stakeholders can analyse the datasets together. Based on the interaction during the development of the methods and tools in the TOICA project, engagement and critical understanding is significantly improved.

Here also mention the identified benefits so far, and emphasize again on further potentials and contributions in engineering design.

Benefit include

- Ability to represent many design alternatives

- Evaluated simultaneously for many variables

- Has aggregated information, where dynamic navigation in resolution and underlying assumptions can be done interactively

- Analysis supported by ability to search for better solution areas.

- Describe the logic -Disciplinary development via computational tools

- Ability to combine disciplines (multi-physics)

- Ability to process large amount of data (computational power..)

- Ability to automatically generate the necessary designs and their variation (design automation, KBE, ...)

- Differentiation in business by tailoring and customizing products and services -> more variations and aspects ageing

Enabling means to support decision making:

- Impact of component performance to global performance

- Identify meaningful trade-offs between requirements and design parameters

- Understand the design space and find answers to the questions:

o What are the important parameters?

o How the requirements compete?

o Where are the most promising solutions?

- Manage risk

For further work there are several directions of interest. One is to further strengthen the interaction with different scientific communities, such as the engineering disciplines, computer science, visual analytics communities, mathematics and cognitive decision making specialists. 
Secondly, already in its current state of maturity, there is an exploitation track to explore. The needs and contexts prevailing in decision making situations during product development can already now benefit from the ability to bring in complex data sets, link them to high level objectives and increase interaction with specialists. There is a route to more clearly understand, demonstrate and implement support for decision making in complex product and production development situations. The ability to represent and characterize architectures via internal and external dependencies is a promising area, where research is needed to enhance representation, definition and evaluation methods and tools. It is likely that the actual decision making process throughout a development project will continue to develop new practices. One of which is the facilitation of more interactive, visual and analytical tools.

\section{Acknowledgements}

The research leading to these results has received funding from the European Seventh Framework Programme (FP7/2007-2013) under grant agreement $\mathrm{n}^{\circ} 604981$, TOICA (www.toica-fp7.eu).

\section{REFERENCES}

[1] T. Kipouros, O. Isaksson, Integrating value assessment into the computational engineering design cycle, in OPT-i 2014: International Conference on Engineering and Applied Sciences Optimization, Kos Island, Greece, Paper 3620, 2014.

[2] O. Isaksson, M. Kossmann, M. Bertoni, H. Eres, A. Monceaux, A. Bertoni, S. Wiseall, X. Zhang, Value-driven design: a methodology to link expectations to technical requirements in the extended enterprise, in INCOSE International Symposium, Philadelphia, PA, 2013.

[3] J.T. Oden, T. Belytschko, I. Babuska, T.J.R. Hughes, Research directions in computational mechanics. Computer Methods in Applied Mechanics and Engineering, 192, 913922, 2003.

[4] R.C Basole, A. Qamar, H. Park, C.J.J. Paredis and L.F. McGinnis, "Visual analytics for early-phase complex engineered system design support,” IEEE Computer Graphics and Application, vol. 35, pp. 41-51, 2015.

[5] X. Yan, M. Qiao, J. Li, T.W. Simpson, G.M. Stump and X. Zhang, “A work-centered visual analytics model to support engineering design with interactive visualization and data-mining,” in 45th Hawaii International Conference on System Sciences, IEEE, pp. 1845-1854, 2012.

[6] M.J. Woodruff, P.M. Reed and T.W. Simpson, "Many objective visual analytics: rethinking the design of complex engineered systems," Struct Multidisc Optim, vol. 48, pp. 201-219, 2013.

[7] A. Inselberg, "Parallel coordinates: visual multidimensional geometry and its applications,” Springer, New York, 2009.

[8] D-H. Ham, "The state of the art of visual analytics," Springer Proceedings in Physics, vol. 135, pp. 213-222, 2010.

[9] S.D. Scott, N. Lesh and G.W. Klau, "Investigating human-computer optimization," Mitsubishi Electric Research Laboratories, TR2001-39, 2001. 
[10] T. Kipouros, "Human-in-the-loop computational engineering design," in EVOLVE International Conference, 2014.

[11] A. Lewis and T. Kipouros, "Methods of interactive optimisation in engineering design," in 13th International Conference on Computer Applications, 2015.

[12] H.A. Nguyen, D. Abramson, T. Kipouros, A. Janke and G. Galloway, "Workways: interacting with scientific workflows," Concurrency and Computation: Practice and Experience, 2015. DOI: 10.1002/cpe.3525.

[13] J. Heinrich, "Visualization techniques for parallel coordinates," PhD Thesis, University of Stuttgart, 2013.

[14] M. H. Eres, M. Bertoni, M. Kossmann, J. P. Scanlan, "Mapping customer needs to engineering characteristics: an aerospace perspective for conceptual design". Journal of Engineering Design, DOI: 10.1080/09544828.2014.903387, 2014.

[15] D.A. Keim, J. Kohlhammer, G. Ellis, F. Mansmann (eds) (2010) "Mastering the information age - solving problems with visual analytics" Eurographics, available at http://www.vismaster.eu/wp-content/uploads/2010/11/VisMaster-book-lowres.pdf

[16] A. Bertoni, M. Bertoni, O. Isaksson, , "Communicating the Value of PSS Design Alternatives using Color-Coded CAD Models", in J. Hesselbach and C. Herrmann (eds.), Functional Thinking for Value Creation: Proceedings of the 3rd CIRP International Conference on Industrial Product Service Systems, Technische Universität Braunschweig, Braunschweig, Germany, May 5th - 6th, 2011

[17] D. Raudberget, C. Levandowski, O., Isaksson, T. Kipouros, H. Johannesson, J. Clarkson, "Modelling and assessing platform architectures in pre-embodiment phases through setbased evaluation and change propagation”, Journal of Aerospace Operations, vol. 3, no. 3,4, pp. 203-221, 2015

[18] P. Clarkson, C. Simons and C. Eckert, "Predicting change propagation in complex design,” J Mech Des 126, (2004), pp. 788-797 